\title{
Some Basic Concepts of Algebraic Quantum Theory
}

\author{
J. E. Roberts and G. Ronpstorfa \\ II. Institut für Theoretische Physik der Universität Hamburg
}

Received October 14, 1968

\begin{abstract}
After briefly putting algebraic quantum theory into the context of a probabilistic interpretation with emphasis on local measurements, certain general features of the theory are examined. Sectors are defined and shown to be the components of the pure state space in the norm topology. Transition probabilities are defined by a simple algebraic formula and it is shown how superpositions of pure states may be defined. With the aid of these results, symmetries are characterized and the connexion with Wigner's Theorem is established.
\end{abstract}

\section{Introduction}

The predictions of quantum theory were quickly realized to be probabilistic in nature. These predictions do not fall within the scope of classical probability theory but they can be accommodated within a noncommutative probability theory. The probabilistic formulation of quantum theory owes much to the pioneering work of von NEUManN [1] but the clearest account of the essentials has been provided by MAckey [2]. Here the states and the observables are treated as the primary entities of the theory and a probability measure is assigned to each pair $(\omega, A)$ consisting of a state $\omega$ and an observable $A$.

The algebraic approach to quantum theory with its stress on the $C^{*}$-algebra of bounded observables was initiated by SEGAL [3] and was realized by ARAKI [4], HAAG and KASTLER [5] to provide a useful tool for understanding local quantum field theory. The relationship of algebraic quantum theory to Mackey's axioms has recently been clarified by Plymen [6] using the concept of a $\Sigma^{*}$-algebra introduced by DAviEs [7]. As Davies showed, it is always possible to embed an abstract $C^{*}$-algebra $\mathfrak{A}$ in a canonical way in a $\Sigma^{*}$-algebra $\mathfrak{A}^{\sim}$ so that any state of $\mathfrak{A}$ has a unique extension to a $\sigma$-state on $\mathfrak{A} \sim . \mathfrak{A} \sim$ is called the $\sigma$-envelope of $\mathfrak{A}$. In classical statistical mechanics if $\mathfrak{A}$ is chosen to be the $C^{*}$-algebra of continuous functions on a compact subset of phase space, $\mathfrak{A}^{\sim}$ may be identified with the $\Sigma^{*}$-algebra of bounded Borel functions on that subset. In section 2 , we discuss this relationship between $C^{*}$-algebras and $\Sigma^{*}$-algebras in the light of local measurements and show how unbounded observables fit naturally into the scheme.

Regarding the probabilistic formulation of quantum theory as fundamental, we take the view that a symmetry of a physical system is

Commun. math. Phys., Vol.11 
most properly defined as a transformation leaving invariant the underlying probability measures. In Section 3, we discuss the implications of this assumption in a $C^{*}$-realization of a physical system. Our main result here is that any transformation of the states induced by a symmetry is an isometry with respect to the metric induced by the norm on $\mathfrak{U}^{\prime}$, the dual of $\mathfrak{A}$.

Before establishing the connexion with the Wigner-Bargmann [8 and 9] analysis of symmetries, we need to define sectors, establish some of their properties and also give an algebraic definition of the transition probability between two pure states. This forms the material of Section 4 and allows us to formulate the superposition principle in Section $\mathbf{5}$ and show its invariance under symmetry transformations.

We can now show in Section 6 that transition probabilities are left invariant by a symmetry whilst the sectors are permuted by it. Thus, applying Wigner's Theorem in the extended version due to BARGMaNN [9], we can infer the existence of a unitary or antiunitary mapping implementing the mapping from some initial sector to the corresponding final sector. This allows us to rederive a result of KADIson [10] that a symmetry induces a Jordan isomorphism of the $C^{*}$-algebra. We conclude with a necessary and sufficient condition for a transformation of the pure states to be induced by a symmetry.

For the convenience of the reader, we add a few remarks on the terminology and notation used. If $\alpha$ is a map from a set $X$ to a set $Y$, then for any subset $M$ of $Y, \alpha^{-1}(M)$ denotes the inverse image of $M$ in $X$. If $\alpha$ is $1-1$ and onto it will be called a bijection. If $\alpha: X \rightarrow Y$ and $\beta: Y \rightarrow Z$ are two successive maps, $\beta \circ \alpha$ denotes the composed map $X \rightarrow Z$. $\boldsymbol{R}$ and $\boldsymbol{C}$ denote the fields of real and complex numbers respectively. A function $f: \boldsymbol{R} \rightarrow \boldsymbol{R}$ is called a (real-valued) Borel function, if $f^{-1}(B)$ is a Borel set of $\boldsymbol{R}$ for any Borel set $B \subset \boldsymbol{R}$. A probability measure $\mu$ on $\boldsymbol{R}$ is a non-negative Borel measure such that $\mu(\boldsymbol{R})=1$.

By a $C^{*}$-algebra $\mathfrak{A}$, we shall always mean an abstractly defined $C^{*}$-algebra with unit, denoted by $\mathrm{I}$. For details the reader is referred to Dixmier's book [11]. If $\Omega$ is a compact Hausdorff space, then $C(\Omega)$ denotes the $C^{*}$-algebra of continuous functions $\Omega \rightarrow C$. The spectrum of $A \in \mathfrak{A}$ is the set of complex numbers $\lambda$ such that $A-\lambda I$ has no inverse in $\mathfrak{A}$ and is denoted by $\operatorname{Sp} A . \operatorname{Sp} A$ is always compact and if $A$ is Hermitian then $\operatorname{Sp} A$ is real. We shall denote by $\mathfrak{A}_{h}$ the Jordan algebra of Hermitian elements of the $C^{*}$-algebra $\mathfrak{A}$. A Jordan homomorphism is a ${ }^{*}$-preserving linear map of a $C^{*}$-algebra $\mathfrak{A}$ into a $C^{*}$-algebra $\mathfrak{B}$ such that $\phi\left(A^{2}\right)=\phi(A)^{2}$ for each $A \in \mathfrak{A}$. A bijective Jordan homomorphism is called a Jordan isomorphism.

By a representation $\pi$ of $\mathfrak{A}$, we mean a representation by bounded operators on some complex Hilbert space $\mathscr{H}_{\pi}$. $\hat{\tau}$ denotes the equivalence 
class of unitarily equivalent representations to which $\pi$ belongs. The set of equivalence classes of irreducible representations is denoted by $\hat{\mathfrak{A}}$ and called the spectrum of $\mathfrak{A} . S(\mathfrak{Q})$ denotes the set of states of $\mathfrak{A}$, i.e. the set of positive linear functionals of norm 1. The extreme points of the convex set $S(\mathfrak{A})$ are called pure states and the set of pure states is denoted by $P(\mathfrak{U})$. If $\omega \in S(\mathfrak{A}), \pi_{\omega}$ denotes the canonical cyclic representation in the Hilbert space $\mathscr{H}_{\omega}$ with cyclic unit vector $x_{\omega}$ for which $\omega(A)=\left(x_{\omega}, \pi_{\omega}(A) x_{\omega}\right)$ for all $A \in \mathfrak{A}[11 ; 2.4 .4]$. This is often referred to as the Gelfand-Naimark-Segal construction. $\pi_{\omega}$ is irreducible if and only if $\omega$ is pure $[11 ; 2.5 .4]$ and we thus have a canonical mapping $\omega \longmapsto \hat{\pi}_{\omega}: P(\mathfrak{Q}) \rightarrow \hat{\mathfrak{A}}$. A vector state on $\mathfrak{A}$ in the representation $\pi$ is a state $\omega_{x} \in S(\mathfrak{Q})$ such that $\omega_{x}(A)=(x, \pi(A) x)$ for all $A \in \mathfrak{A}$ and some unit vector $x \in \mathscr{H}_{\pi}$. A full set of states [10] is a convex subset $\mathscr{S} \subset S(\mathfrak{A})$ such that $A \geqq 0$ in $\mathfrak{A}$ if $\omega(A) \geqq 0$ for all $\omega \in \mathscr{S}$. The norm topology on $S(\mathfrak{A})$ is that induced by the dual norm on $\mathfrak{A}^{\prime}$ and is derived from the metric $\delta\left(\omega, \omega^{\prime}\right)=\left\|\omega-\omega^{\prime}\right\|=\sup _{\|\boldsymbol{A}\| \leqq 1}\left|\omega(A)-\omega^{\prime}(A)\right|$. The $w^{*}$-topology on $S(\mathfrak{Q})$ is the coarsest topology making the map $\omega \longmapsto \omega(A)$ continuous for each $A \in \mathfrak{A}$.

We shall also use the concept of a spectral measure, but only in the special case of a spectral measure over $\boldsymbol{R}$ with values in a $\Sigma^{*}$-algebra $\mathfrak{A}$. In this case we mean a map $B \mapsto E(B)$ from the Borel sets of $\boldsymbol{R}$ into the projections of $\mathfrak{A}$ such that $E(\emptyset)=0, E(\boldsymbol{R})=\mathrm{I}$ and $E\left(B_{1} \cap B_{2}\right)$ $=E\left(B_{1}\right) E\left(B_{2}\right)$ for every pair of Borel sets $B_{1}$ and $B_{2}$ and which is countably additive with respect to the weak operator topology of some faithful $\sigma$-representation of $\mathfrak{A}[7]$.

\section{Local Measurement and $C^{*}$-Realizations}

We choose the same starting point as MACKEY [2] and suppose that if $\mathscr{S}$ is any set of states of a physical system and $\mathscr{A}$ any set of observables of that system, then there is a map $\mu$ from $\mathscr{S} \times \mathscr{A}$ into the set of probability measures on $\boldsymbol{R}$. The physical interpretation of $\mu(\omega, A)$ is that it predicts the probability distribution of measured values of $A$ when the system is in the state $\omega$. The expectation value of $A$ in the state $\omega, \omega(A)$, is simply the first moment of $\mu(\omega, A): \omega(A)=\int \lambda \mu(\omega, A)(d \lambda)$. This expectation value will not necessarily exist if $A$ is unbounded.

If we wish to give a mathematical model for a physical system, we must identify $\mathscr{S}$ and $\mathscr{A}$ with certain abstract sets in such a way that there is a natural probability measure associated with each pair $(\omega, A)$. Formally, this may be achieved by mapping $\mathscr{S}$ and $\mathscr{A}$ into such sets. This will then be called a realization of $(\mathscr{S}, \mathscr{A}, \mu)$, and we shall be concerned here with three types of realizations. 
Definition 2.1. Let $\mathfrak{A}$ be a $C^{*}$-algebra and $S(\mathfrak{A})$ the set of states of 2. A $C^{*}$-realization of $(\mathscr{S}, \mathscr{A}, \mu)$ consists of maps $\omega \longmapsto \hat{\omega}: \mathscr{S} \rightarrow S(\mathfrak{A})$ and $A \longmapsto \hat{A}: \mathscr{A} \rightarrow \mathfrak{A}_{h}$ such that $\mu(\omega, A)=\hat{\mu}(\hat{\omega}, \hat{A})$, where $\hat{\mu}(\hat{\omega}, \hat{A})$ is the unique probability measure on $\boldsymbol{R}$ with support in $\operatorname{Sp} \hat{A}$ such that $\hat{\omega}(g(\hat{A}))$ $=\int g(\lambda) \hat{\mu}(\hat{\omega}, \hat{A})(d \lambda)$ for all $g \in C(\operatorname{Sp} \hat{A})^{1}$. A $C^{*}$-realization is called a $\Sigma^{*}$-realization if $\mathfrak{A}$ is a $\Sigma^{*}$-algebra and if the image of $\mathscr{S}$ in $S(\mathfrak{U})$ consists of $\sigma$-states. We have a $\Sigma^{*}$-spectral realization of $(\mathscr{S}, \mathscr{A}, \mu)$, if $\mathfrak{A}$ is a $\Sigma^{*}$-algebra, $\mathscr{S}$ is mapped into $\sigma$-states on $\mathfrak{A}$, and if there is a map $A \longmapsto E_{A}$ from $\mathscr{A}$ into the spectral measures over $\boldsymbol{R}$ with values in $\mathfrak{A}$ such that $\mu(\omega, A)(B)=\hat{\omega}\left(E_{A}(B)\right)$ for every Borel set $B \subset \boldsymbol{R}$.

It is clear that $(\mathscr{S}, \mathscr{A}, \mu)$ cannot have a $C^{*}$ - or $\Sigma^{*}$-realization if $\mathscr{A}$ contains unbounded operators. The advantage of $\Sigma^{*}$-spectral realizations is that they are possible even when $\mathscr{A}$ contains unbounded observables. Now any $C^{*}$-realization determines a canonical $\Sigma^{*}$-realization, since a $C^{*}$-algebra $\mathfrak{A}$ may be embedded into its $\sigma$-envelope $\mathfrak{A} \sim$ in such a way that any state of $\mathfrak{A}$ has a unique extension to a $\sigma$-state of $\mathfrak{A} \sim[7$; Theorem 3.1]. Moreover, any $\Sigma^{*}$-realization determines a $\Sigma^{*}$-spectral realization, since the Hermitian elements of a $\Sigma^{*}$-algebra are in $1-1$ correspondence with the compact spectral measures over $\boldsymbol{R}$ with values in $\mathfrak{A}[6 ;$ Lemma 2.5]. If $E_{\hat{A}}$ corresponds to $\hat{A} \in \mathfrak{A}_{h}$ it is easy to verify that $\hat{\mu}(\hat{\omega}, \hat{A})$ $=\hat{\omega} \circ E_{\hat{A}}$.

It is useful to introduce an abstract version of the correspondence $\hat{A} \leftrightarrow E_{\hat{A}}$ in the context of a triplet $(\mathscr{S}, \mathscr{A}, \mu)$. We recall that a question [2], $Q \in \mathscr{A}$, is an observable such that $\mu(\omega, Q)$ is concentrated on $\{0,1\}$ for all $\omega \in \mathscr{S}$.

Definition 2.2. An observable $A \in \mathscr{A}$ is said to have a spectral resolution in $\mathscr{A}$, if there is a question-valued measure [2], $Q_{A}$, over $\boldsymbol{R}$ such that $\omega \circ Q_{A}=\mu(\omega, A)$ for all $\omega \in \mathscr{S}$.

We now turn to a description of local measurements. To measure a particular observable, we need a measuring device, whose specification will be supposed to include the spatial extension of the apparatus at any time $t$ in some frame. Thus, in Minkowski space, the associated region may be thought of as a tube. The intersection of such a tube with a hyperplane $t=$ const. will be called a local section of Minkowski space. Every measuring apparatus will be assumed to possess a pointer, or similar device, whose position indicates the result of the experiment. We assume that any series of measurements can be reduced to single measurements which consist in reading the position of the pointer at a particular instant of time. This instant of time defines a local section $s(m)$ associated with each measurement $m$, and $s(m)$ is compact as a consequence of the local nature of $m$.

1 The existence of $\hat{\mu}$ is guaranteed by applying Bochner's Theorem to the function $F^{\prime}(t)=\widehat{\omega}\left(e^{i t \hat{A}}\right) . \hat{\mu}$ is just the state on $C(\operatorname{Sp} \hat{A})$ induced by $\hat{\omega}$. 
We assume that given any state $\omega \in \mathscr{S}$, there is a probability measure $\mu(\omega, m)$ on $s(m)$ relative to the natural Borel structure of $s(m)$. The measure $\mu(\omega, m)$ determines the probability distribution over $s(m)$ of the position of the pointer when the system is in the state $\omega$. Let $S(m)$ denote the joint support of the measures $\mu(\omega, m)$ as $\omega$ varies in $\mathscr{S}$. Thus $x \in S(m)$ if and only if, given an open neighbourhood $N_{x}$ of $x$ in $s(m)$, there exists an $\omega \in \mathscr{S}$ with $\mu(\omega, m)\left(N_{x}\right)>0 . S(m)$ is a compact subset of $s(m)$, and we shall assume that $S(m)$ is homeomorphic to a compact subset of $\boldsymbol{R}$. We are here ignoring, for example, the possibility of twoor three-dimensional graphical representations of measurements ${ }^{2}$.

Before associating an observable with a measurement $m$, we must provide the pointer with a scale over which it moves. We do this abstractly by specifying a Borel function $f: S(m) \rightarrow \boldsymbol{R}$. Different Borel functions give different scales and determine different observables. In this way, we get a mapping $f \mapsto m(f)$ from the Borel functions on $S(m)$ into $\mathscr{A}$ such that $\mu(\omega, m(f))=f_{*} \mu(\omega, m)$, where $f_{*} \mu$ denotes the probability measure on $\boldsymbol{R}$ defined by $\left(f_{*} \mu\right)(B)=\mu\left(f^{-1}(B)\right)$ for all Borel sets $B$ of $\boldsymbol{R}$.

In most cases, it is a reasonable idealization to assume that the measuring apparatus of a measurement $m$ disturbs the system under observation only within some compact subset $d(m)$ of Minkowski space. This set must be distinguished from $s(m)$, because it would neither be reasonable to assume that $d(m)$ was contained in a time-hyperplane, nor even that the time at which the pointer was read corresponded to a time when the apparatus was in effective interaction with the system.

Let $\mathcal{O}$ be a bounded open subset of Minkowski space and let ob( $\mathcal{O})$ denote the set of observables of the form $m(f)$, where $m$ is any measurement with $d(m) \subset \mathcal{O}$ and $f$ is any Borel function, $f: S(m) \rightarrow \boldsymbol{R}$. Let bob $(\mathcal{O})$ and $\operatorname{cob}(\mathcal{O})$ denote the subsets of $o b(\mathcal{O})$ determined by restricting $f$ to be a bounded Borel function or a continuous function respectively.

Proposition 2.3. Every $m(f) \in \mathrm{ob}(\mathcal{O})$ has a spectral resolution with values in bob $(\mathcal{O})$.

Proof. Let $B$ be a Borel set of $\boldsymbol{R}$, then $f^{-1}(B)$ is a Borel set of $S(m)$, and if $\chi_{f^{-1}(B)}$ denotes its characteristic function, $m\left(\chi_{f^{-1}(B)}\right)$ is a question in bob $(\mathcal{O})$. If we now set $Q_{m(f)}(B)=m\left(\chi_{f^{-1}(B)}\right)$, then $\omega \circ Q_{m(f)}(B)$ $=\omega\left(m\left(\chi_{f^{-1}(B)}\right)\right)=\mu(\omega, m(f))(B)$. Hence $\omega \circ Q_{m(f)}=\mu(\omega, m(f))$ for all $\omega \in \mathscr{S}$. This shows that $B \mapsto Q_{m(f)}(B)$ is a question-valued measure and further that it is the spectral resolution of $m(f)$.

${ }^{2}$ Restricting $S(m)$ to be homeomorphic to a compact subset of $\boldsymbol{R}$ has no deep significance. If we wished to relax this condition, we ought, in the same spirit to allows observables $A$ for which $\mu(\omega, A)$ is a probability measure on $\boldsymbol{R}^{n}, n \geqq 1$. 
We now assume that we have a $C^{*}$-realization of $(\mathscr{P}, \operatorname{cob}(\mathscr{O}), \mu)$ in a $C^{*}$-algebra $C^{*}(\mathcal{O})$ defined by mappings $m(f) \longmapsto \hat{m}(f)$ and $\omega \longmapsto \hat{\omega}$, and that $\hat{\mathscr{S}}=\{\hat{\omega}: \omega \in \mathscr{S}\}$ is a full set of states of $C^{*}(\mathcal{O})$.

Proposition 2.4. Let $m(f) \in \operatorname{cob}(\mathcal{O})$, then

a) $\operatorname{Sp} \hat{m}(f)=f(S(m))$.

b) $g(\hat{m}(f))=\hat{m}(g \circ f)$ for $g \in C(\operatorname{Sp} \hat{m}(f))_{h}$.

c) $\left\{\hat{m}(f): f \in C(S(m))_{h}\right\}$ is a Jordan subalgebra of $C^{*}(\mathcal{O})$ isomorphic to $C(S(m))_{h}$ and $f \mapsto \hat{m}(f)$ realizes this isomorphism.

Proof. a) Since $\mathrm{Sp} \hat{m}(f)$ supports the measures associated with $\hat{m}(f)$ in $C^{*}(\mathcal{O}), f(S(m))<\operatorname{Sp} \hat{m}(f)$. Let $g: \operatorname{Sp} \hat{m}(f) \rightarrow \boldsymbol{R}$ be any continuous function such that $g \circ f(S(m))=\{0\}$. Then, making free use of the functional calculus in $C^{*}$-algebras $[11 ; 1.5]$,

$$
\hat{\omega}(g(\hat{m}(f)))=\int g(\lambda) \mu(\omega, m(f))(d \lambda)=0
$$

for all $\omega \in \mathscr{S}$. Hence $g(\hat{m}(f))=0$, as $\mathscr{S}$, being full, separates $C^{*}(\mathcal{O})$. But $f(S(m))$ is closed in Sp $\hat{m}(f)$, and it follows by URYsoHN's Lemma [12; $\left.\S 4, \mathrm{~N}^{0} 1\right]$ that $f(S(m))=\mathrm{Sp} \hat{m}(f)$.

b) $\hat{\omega}(\hat{m}(g \circ f))=\int \lambda(g \circ f)_{*} \mu(\omega, m)(d \lambda)=\int g(\lambda) \mu(\omega, m(f))(d \lambda)$. Hence $\hat{\omega}(\hat{m}(g \circ f))=\hat{\omega}(g(\hat{m}(f))$ for all $\hat{\omega} \in \hat{\mathscr{S}}$. Thus $\hat{m}(g \circ f)=g(\hat{m}(f))$.

c) By hypothesis, there is a homeomorphism $h: S(m) \rightarrow I \subset \boldsymbol{R}$. From a), $\operatorname{Sp}(\hat{m}(h))=h(S(m))=I$. Now $g \longmapsto g(\hat{m}(h))$ is an isomorphism of $C(I)_{h}$ onto a Jordan subalgebra of $C^{*}(\mathcal{O})[11 ; 1.5 .1]$. Hence

$$
f \mapsto f \circ h^{-1}(\hat{m}(h))=\hat{m}(f)
$$

is, by b), an isomorphism of $C(S(m))_{h}$ onto a Jordan subalgebra of $C^{*}(\mathcal{O})$.

Theorem 2.5. The $C^{*}$-realization of $(\mathscr{S}, \operatorname{cob}(\mathcal{O}), \mu)$ in $C^{*}(\mathcal{O})$ may be extended to a $\Sigma^{*}$-realization of $(\mathscr{S}, \operatorname{bob}(\mathcal{O}), \mu)$ in $\Sigma^{*}(\mathcal{O})$, where $\Sigma^{*}(\mathcal{O})$ denotes the $\sigma$-envelope of $C^{*}(\mathcal{O})$, and to a $\Sigma^{*}$-spectral realization of $(\mathscr{S}, \mathrm{ob}(\mathcal{O}), \mu)$ in $\Sigma^{*}(\mathcal{O})$.

Proof. By Proposition 2.4, $f \longrightarrow \hat{m}(f)$ is an injective morphism $C(S(m)) \rightarrow C^{*}(\mathcal{O})$, and since $C^{*}(\mathcal{O})$ may be regarded as a subalgebra of $\Sigma^{*}(\mathcal{O})$, we equally have an injective morphism $C(S(m)) \rightarrow \Sigma^{*}(\mathcal{O})$. Picking a faithful $\sigma$-representation of $\Sigma^{*}(\mathcal{O})$ and applying a result of DAvIES [7; Lemma 2.3], we get we get a morphism $B\{S(m)\} \rightarrow \Sigma^{*}(\mathcal{O})$, where $B\{S(m)\}$ denotes the $\Sigma^{*}$-algebra of complex Borel functions on $S(m)$. Identifying $\{m(f): f: S(m) \rightarrow \boldsymbol{R}$ is bounded Borel $\}$ with the Hermitian elements of $B\{S(m)\}$ in the obvious way, we may extend $m(f) \mapsto \hat{m}(f)$ to bounded Borel functions. Doing this for all $m$, we get a mapping bob $(\mathcal{O}) \rightarrow \Sigma^{*}(\mathcal{O})$. The states of $C^{*}(\mathcal{O})$ may be identified with the $\sigma$-states of $\Sigma^{*}(\mathcal{O})$ and $\mu(\omega, m(f))=\hat{\mu}(\hat{\omega}, \hat{m}(f))$ for all bounded Borel functions $f: S(m) \rightarrow \boldsymbol{R}$. We have now constructed a $\Sigma^{*}$-realization of $(\mathscr{S}, \operatorname{bob}(\mathcal{O}), \mu)$ extending the $C^{*}$-realization of $(\mathscr{S}, \operatorname{cob}(\mathcal{O}), \mu)$ as required. The extension to a $\Sigma^{*}$-spectral realization of $(\mathscr{S}$, ob $(\mathcal{O}), \mu)$ in $\Sigma^{*}(\mathcal{O})$ follows at 
once by taking the $\Sigma^{*}$-spectral realization of $(\mathscr{S}$, bob $(\mathcal{O}), \mu)$ determined by its $\Sigma *$-realization and applying Proposition 2.3 to extend from $\operatorname{bob}(\mathcal{O})$ to $\operatorname{ob}(\mathcal{O})$.

The results of this section show that, as far as local measurements go, there is a natural connexion between the $C^{*}$-realizations, $\Sigma^{*}$-realizations and $\Sigma *$-spectral realizations. Which realization is chosen is largely a matter of taste or convenience with respect to the particular problem under consideration. It is perhaps worth remarking here that since the von Neumann algebras so far used in physics are von Neumann algebras in separable Hilbert spaces, it is of no consequence if they are regarded as $\Sigma^{*}$-algebras since every $\sigma$-state is automatically normal. Note also Kadison [7; Appendix, Theorem A].

It is usual to regard as observables quantities which cannot strictly speaking be measured by a local measurement. Thus the $C^{*}$-algebras of strictly local observables are embedded in a $C^{*}$-algebra of quasilocal observables [5] and even global quantities such as total charge and total energy are reckoned as observables. It is not part of the aims of this paper to examine the relationship between local and global observables, and the rest of this paper will be devoted to describing certain consequences of assuming that a physical system may be adequately described by a $C^{*}$-realization of certain of its observables.

\section{Symmetries}

A symmetry of a physical system is intuitively a transformation of the system leaving all physically significant features invariant. We have chosen to introduce quantum theory in terms of the set of states $\mathscr{S}$ of a physical system, the set of observables $\mathscr{A}$ of that system and a probability measure $\mu(\omega, A)$ defined for each pair $\omega \in \mathscr{S}$ and $A \in \mathscr{A}$. In this approach, we naturally define a symmetry to be a pair of bijections $\alpha: \mathscr{S} \rightarrow \mathscr{S}$ and $\bar{\alpha}: \mathscr{A} \rightarrow \mathscr{A}$ such that $\mu(\alpha(\omega), \bar{\alpha}(A))=\mu(\omega, A)$, for all $\omega \in \mathscr{S}$ and $A \in \mathscr{A}$. Thus the a priori physically significant features are the notions of state and observable of the system and the probability measure assigned to a pair $(\omega, A)$. It now follows that $\omega(A)=\alpha(\omega)(\bar{\alpha}(A))$ in the sense that, if either side of the equation exists, then so does the other and equality holds. Thus expectation values are preserved by a symmetry.

In the course of this paper, we shall analyse the possible forms of a symmetry in the $C^{*}$-algebra context. To fix ideas, we suppose that the quasilocal bounded observables of a physical system can be realized, in the sense of the previous section, by the Hermitian elements $\mathfrak{A}_{h}$ of a $C^{*}$-algebra $\mathfrak{A}$ and $\mathscr{S}$ by a full set of states of $\mathfrak{A}$. We shall no longer distinguish between $A$ and $\hat{A}$ or $\omega$ and $\hat{\omega}$ for the remainder of this paper. 
Now if $A \in \mathscr{A}$ is bounded, then so is $\bar{\alpha}(A)$ and it is a reasonable requirement that if $A$ is a quasilocal bounded observable then so is $\bar{\alpha}(A)$. Thus we assume that $\bar{\alpha}$ induces a mapping $\mathfrak{A}_{h} \rightarrow \mathfrak{A}_{h}$, which we again denote by $\bar{\alpha}$.

If $A, B \in \mathfrak{A}_{h}$, we set $\bar{\alpha}(A+i B)=\bar{\alpha}(A)+i \bar{\alpha}(B)$ and thus extend $\bar{\alpha}$ to a mapping $\bar{\alpha}: \mathfrak{A} \rightarrow \mathfrak{A}$.

Proposition 3.1. If $\alpha: \mathscr{S} \rightarrow \mathscr{S}$ and $\bar{\alpha}: \mathfrak{A} \rightarrow \mathfrak{A}$ arise from a symmetry then $\alpha$ is affine and $w^{*}$-continuous and $\bar{\alpha}$ is linear.

Proof. If $\lambda, \mu \in \boldsymbol{R}, \lambda, \mu \geqq 0$ and $\lambda+\mu=1$, and if $\omega_{1}, \omega_{2} \in \mathscr{S}$ then $\lambda \omega_{1}+\mu \omega_{2} \in \mathscr{S}$, since $\mathscr{S}$ is full. Now

$\alpha\left(\lambda \omega_{1}+\mu \omega_{2}\right)(A)=\left(\lambda \omega_{1}+\mu \omega_{2}\right)\left(\bar{\alpha}^{-1}(A)\right)=\lambda\left(\alpha \omega_{1}\right)(A)+\mu\left(\alpha \omega_{2}\right)(A)$ for all $A \in \mathfrak{A}$. Hence $\alpha\left(\lambda \omega_{1}+\mu \omega_{2}\right)=\lambda \alpha\left(\omega_{1}\right)+\mu \alpha\left(\omega_{2}\right)$, i.e. $\alpha$ is affine. Now the $w^{*}$-topology on $\mathscr{S}$ is the coarsest topology on $\mathscr{S}$ making the mappings $\omega \longmapsto \omega(A)$ continuous for each $A \in \mathfrak{A}$. However $\alpha(\omega)(A)$ $=\omega\left(\bar{\alpha}^{-1}(A)\right)$ so that the mapping $\omega \longmapsto \alpha(\omega)(A)$ is $w^{*}$-continuous for each $A \in \mathcal{A}$. Hence $\alpha$ is $w^{*}$-continuous $[13 ; \S 2$, No. 3]. Now if $\lambda, \mu \in \boldsymbol{C}$, $\omega(\bar{\alpha}(\lambda A+\mu B))=\alpha^{-1}(\omega)(\lambda A+\mu B)=\omega(\lambda \bar{\alpha}(A)+\mu \bar{\alpha}(B))$ for all $\omega \in \mathscr{S}$. But $\mathscr{S}$ is a full set of states, so that $\bar{\alpha}(\lambda A+\mu B)=\lambda \bar{\alpha}(A)$ $+\mu \bar{\alpha}(B)$ and $\bar{\alpha}$ is linear.

Now Kadison [10; Theorem 2.2] shows that a convex subset of $S(\mathfrak{Z})$ is full if and only if it is $w^{*}$-dense in $S(\mathfrak{Q})$. Hence we may extend $\alpha$ by continuity to a mapping $\alpha: S(\mathfrak{H}) \rightarrow S(\mathfrak{U})$ such that $\alpha(\omega)(\bar{\alpha}(A))$ $=\omega(A)$ for all $A \in \mathfrak{U}$ and $\omega \in S(\mathfrak{Q}) . \alpha$ is now a $w^{*}$-homeomorphism of $S(\mathfrak{U})$. Furthermore, we may prove, as in Proposition 3.1, that $\bar{\alpha}$ is weakly continuous, and $\alpha$ is clearly just the restriction of the dual of $\bar{\alpha}^{-1}$ to $S(\mathfrak{A})$.

It is possible to start from the apparently weaker hypothesis that a symmetry is determined by a mapping $\alpha: \mathscr{S} \rightarrow \mathscr{S}$ which is affine and a $w^{*}$-homeomorphism, and deduce the existence of the mapping $\bar{\alpha}: \mathfrak{A} \rightarrow \mathfrak{A}[10 ;$ Corollary 4.7]. However, we prefer to adopt a notion of symmetry which has a more immediate physical interpretation and which allows us to simplify the mathematical analysis.

Proposition 3.2. If $\alpha: S(\mathfrak{U}) \rightarrow S(\mathfrak{A})$ arises from a symmetry, and $\omega_{1}, \omega_{2} \in S(\mathfrak{l})$, then $\left\|\alpha\left(\omega_{1}\right)-\alpha\left(\omega_{2}\right)\right\|=\left\|\omega_{1}-\omega_{2}\right\|$. In other words, $\alpha$ is an isometry. Hence

Proof. If $A \in \mathfrak{A}_{h}$, then $\|A\|=\sup \{|\omega(A)|: \omega \in S(\mathfrak{X})\}, \quad[11 ; 2.6 .3]$.

$$
\begin{aligned}
\|\bar{\alpha}(A)\| & =\sup \{|\omega(\bar{\alpha}(A))|: \omega \in S(\mathfrak{A})\} \\
& =\sup \left\{\left|\alpha^{-1}(\omega)(A)\right|: \omega \in S(\mathfrak{R})\right\}=\|A\| .
\end{aligned}
$$

Now $\alpha\left(\omega_{1}\right)-\alpha\left(\omega_{2}\right)$ is Hermitian, so, by $[11 ; 1.2 .6]$,

$$
\begin{aligned}
\| \alpha\left(\omega_{1}\right)- & \alpha\left(\omega_{2}\right) \| \\
& =\sup \left\{\left|\alpha\left(\omega_{1}\right)(A)-\alpha\left(\omega_{2}\right)(A)\right|: A \in \mathfrak{A}_{h},\|A\| \leqq 1\right\} .
\end{aligned}
$$


Hence

$$
\begin{aligned}
\| \alpha\left(\omega_{1}\right)- & \alpha\left(\omega_{2}\right) \| \\
& =\sup \left\{\left|\left(\omega_{1}-\omega_{2}\right)\left(\bar{\alpha}^{-1}(A)\right)\right|: A \in \mathscr{A}_{h},\|A\| \leqq 1\right\} .
\end{aligned}
$$

But $\left\|\bar{\alpha}^{-1}(A)\right\|=\|A\|$ if $A \in \mathfrak{A}_{h}$, so $\left\|\alpha\left(\omega_{1}\right)-\alpha\left(\omega_{2}\right)\right\|=\left\|\omega_{1}-\omega_{2}\right\|$ and $\alpha$ is an isometry.

\section{Sectors and Transition Probabilities}

In the last section, we derived some of the properties of a mapping $\alpha: S(\mathfrak{U}) \rightarrow S(\mathfrak{A})$ arising from a symmetry. Before completing this analysis, we shall show how certain other concepts of elementary quantum theory may be described in the context of $C^{*}$-algebras. We begin by relating the algebraic formalism to the Hilbert space formalism. To fix ideas, we consider a system with commutative discrete superselection rules, so that the Hilbert space of the system, $\mathscr{H}$, decomposes into the direct sum of coherent subspaces $\mathscr{H}=\bigoplus_{i \in I} \mathscr{H}_{i}$. The bounded observables of the system are represented by Hermitian operators on $\mathscr{H}$ leaving each $\mathscr{H}_{i}$ invariant, and the set of bounded observables acts irreducibly in each $\mathscr{H}_{i}$. States represented by unit vectors of $\mathscr{H}$ having non-zero components in more than one $\mathscr{H}_{i}$ are not pure states, since the relative phase is not measurable ${ }^{3}$ Looking at the same situation from the algebraic point of view, we would say that we have a faithful representation $\pi$, say, of a $C^{*}$-algebra of operators on $\mathscr{H}$, which is the direct sum of inequivalent irreducible representations $\pi=\bigoplus_{i \in I} \pi_{i}$. Each unit vector $x \in \mathscr{H}$ defines a state $\omega_{x}$ of $\mathfrak{A}$, where $\omega_{x}(A)=(x, \pi(A) x)$ for all $A \in \mathfrak{A}$. However only those vectors belonging to a particular $\mathscr{H}_{i}$ define a pure state. This leads to our first definition:

Definition 4.1. Let $\pi$ be an irreducible representation of a $C^{*}$-algebra, then the sector associated with $\pi, S_{\hat{\pi}}$, is the set of vector states of the representation $\pi$. Naturally, $S_{\hat{\pi}}$ depends only on $\hat{\pi}$, the unitary equivalence class of $\pi$.

The sectors are the algebraic analogue of the coherent subspaces and our first task will be to investigate their structure.

Proposition 4.2. Let $\omega, \omega^{\prime}$ be pure states of a $C^{*}$-algebra $\mathfrak{A}$, then the following conditions are equivalent.

a) $\omega$ and $\omega^{\prime}$ belong to a common sector of $P(\mathfrak{U})$.

b) There exists a vector $x^{\prime} \in \mathscr{H}_{\omega}$ such that $\omega^{\prime}(A)=\left(x^{\prime}, \pi_{\omega}(A) x^{\prime}\right)$.

c) There exists a unitary element $U \in \mathfrak{A}$ such that $\omega^{\prime}(A)=\omega\left(U^{*} A U\right)$.

d) $\pi_{\omega}$ and $\pi_{\omega^{\prime}}$ are unitarily equivalent.

${ }^{3}$ Such vector states are regarded as being physically unrealizable by authors for whom the term "state" is synonymous with "pure state". 
The sectors form a disjoint covering of $P(\mathfrak{U})$ and $S_{\hat{\pi}}$ is the inverse image of $\hat{\pi}$ under the canonical mapping $P(\mathfrak{U}) \rightarrow \hat{\mathfrak{A}}$.

Proof. If c) holds, $\omega^{\prime}(A)=\left(x^{\prime}, \pi_{\omega}(A) x^{\prime}\right)$ with $x^{\prime}=\pi_{\omega}(U) x_{\omega}$, so c) implies b). If b) holds, $\omega, \omega^{\prime} \in S_{\hat{\pi}_{\omega}}$, so b) implies a). If $\omega \in S_{\hat{\pi}}$, then $\omega(A)=(x, \pi(A) x)$ with $x \in \mathscr{H}_{\pi} . x$ is cyclic as $\pi$ is irreducible, hence $\hat{\pi}_{\omega}=\hat{\pi}$ and a) and d) are equivalent. d) implies c) by a result of GLIMM and KADIson $[14$, and $11 ; 2.8 .6]$. We have now also proved the final statement of the Proposition.

We give one further characterization of a sector which requires two preparatory results. The first, whose proof we omit, is due to GLIMM and Kadison [14].

Lemma 4.3. Let $\mathfrak{A}$ be a $C^{*}$-algebra with identity and let $\omega$ and $\omega^{\prime}$ be pure states of $\mathfrak{A}$, then if $\left\|\omega-\omega^{\prime}\right\|<2$, the representations $\pi_{\omega}$ and $\pi_{\omega^{\prime}}$ are equivalent.

Lemma 4.4. Let $\pi$ be a representation of a $C^{*}$-algebra $\mathfrak{A}$ on $\mathscr{H}_{\pi}$ and let $\pi(\mathfrak{U})$-denote the weak closure of $\pi(\mathfrak{U})$ in $\mathscr{L}\left(\mathscr{H}_{\pi}\right)$. Then the mapping $f \mapsto f \circ \pi$ is a linear isometry from the normal linear functionals on $\pi(\mathfrak{A})^{-}$ into $\mathfrak{A}^{\prime}$, the dual of $\mathfrak{A}$.

Proof. If $f$ is a normal linear functional on $\pi(\mathfrak{U})^{-}$, then $f \circ \pi$ is certainly a continuous linear functional on $\mathfrak{A}$. Further $|f \circ \pi(A)| \leqq\|f\|$ - $\|\pi(A)\| \leqq\|f\|\|A\|$ for all $A \in \mathcal{A}$. Now given $\varepsilon>0$, there exists a $T \in \pi(\mathfrak{U})^{-}$with $\|T\| \leqq 1$ and $|f(T)| \geqq\|f\|-\varepsilon / 2$. Now the ultrastrong and strong topologies agree on the unit ball of $\mathscr{L}(\mathscr{H})$ and by the Kaplansky density theorem [15 and $16 ;$ p. 43-46], there exists an $A \in \mathfrak{A}$ with $\|\pi(A)\| \leqq 1$ and $|f \circ \pi(A)| \geqq\|f\|-\varepsilon$. But, by the uniqueness of the $C^{*}$-norm, $\pi(\mathfrak{U})$ and $\mathfrak{U} / \operatorname{Ker} \pi$ are naturally isometric $[11 ; 1.8 .3]$ so that $\|\pi(A)\|=\inf \{\|B\|: \pi(B)=\pi(A)\}$. Hence we may suppose $A$ has been chosen with $\|A\| \leqq 1+\varepsilon$. Thus

$$
\|f \circ \pi\| \geqq\left|f \circ \pi\left(\frac{A}{1+\varepsilon}\right)\right| \geqq \frac{\|f\|-\varepsilon}{1+\varepsilon} .
$$

But $\varepsilon$ is arbitrary so $\|f \circ \pi\|=\|f\|$ as required.

This Lemma will be applied to the case where $\pi$ is an irreducible representation. The normal linear functionals on $\pi(\mathfrak{A})^{-}=\mathscr{L}\left(\mathscr{H}_{\pi}\right)$ are then the elements of the predual [11; A 23] and may be represented by the operators of trace class and their norm is just the trace norm [16; p. 37-42 and 17].

Proposition 4.5. The sectors are the components of $P(\mathfrak{X})$ in the norm topology.

Proof. Lemma 4.3 and Proposition 4.2 show that the sectors are open. Proposition 4.2 also shows that they form a disjoint covering of $P(\mathfrak{U})$, hence each sector is also closed. It remains to show that they are connected in the norm topology. We can, however, regard $S_{\hat{\jmath}}$ as the set of 
one-dimensional projection operators in $\mathscr{H}_{\pi}$. This set is pathwiseconnected in the trace norm topology by virtue of a simple explicit construction. By Lemma 4.4, the trace norm corresponds to the norm on $S_{\hat{\pi}}$. So $S_{\hat{\pi}}$ is pathwise-connected and hence connected, completing the proof.

This result implies that $\hat{\mathfrak{A}}$ is a discrete space with respect to the corresponding quotient topology under the canonical mapping $P(\mathfrak{A}) \rightarrow \hat{\mathfrak{A}}$.

Returning now to our example of a system with commutative discrete superselection rules, we consider the concept of transition probability. This is customarily defined for pure states only, and if $x$ and $x^{\prime}$ are unit vectors of $\mathscr{H}$ representing pure states, the transition probability between these two states is $\left|\left(x, x^{\prime}\right)\right|^{2}$. This quantity has a simple algebraic interpretation.

Proposition 4.6. Let $\omega_{x}$ and $\omega_{x^{\prime}}$ be the pure states corresponding to the unit vectors $x$ and $x^{\prime}$, then $\left|\left(x, x^{\prime}\right)\right|^{2}=1-\frac{1}{4}\left\|\omega_{x}-\omega_{x^{\prime}}\right\|^{2}$.

Proof. If $x$ and $x^{\prime}$ belong to different coherent subspaces, then $\left|\left(x, x^{\prime}\right)\right|^{2}=0$ and, by Lemma $4.3,\left\|\omega_{x}-\omega_{x^{\prime}}\right\|=2$ as required. If $x$ and $x^{\prime}$ belong to the same coherent subspace $\mathscr{H}_{i}$, say, then, applying Lemma 4.4 to the representation $\pi_{i},\left\|\omega_{x}-\omega_{x^{\prime}}\right\|$ is just the trace norm $\tau(T)$ of $T=x \otimes \bar{x}-x^{\prime} \otimes \bar{x}^{\prime}$. However, $\tau(T)$ is the sum of the moduli of the eigenvalues of $T$ [17], and a routine calculation gives

$$
\left|\left(x, x^{\prime}\right)\right|^{2}=1-\frac{1}{4} \tau(T)^{2}=1-\frac{1}{4}\left\|\omega_{x}-\omega_{x^{\prime}}\right\|^{2} .
$$

This leads us to the following definition:

Definition 4.7. Let $\omega, \omega^{\prime} \in P(\mathfrak{A})$ then $\omega \cdot \omega^{\prime}$, the transition probability between $\omega$ and $\omega^{\prime}$, is given by $\omega \cdot \omega^{\prime}=1-\frac{1}{4}\left\|\omega-\omega^{\prime}\right\|^{2}$.

The temptation to use this formula to define transition probabilities for mixed states should be resisted. For to determine a transition probability from an unknown state $\omega^{\prime}$ into the state $\omega$ operationally, we need to measure an observable so that the state after a (non-selective) measurement is a mixed state, one of whose constituents is the state $\omega$. If the unknown state $\omega^{\prime}$ can be represented by a density matrix $\varrho$, and the pure state by a unit vector $x$, then $P_{x}=x \otimes \bar{x}$ is the projection operator onto the subspace generated by $x$ and is an observable, at least in some approximate sense. The state after the measurement is represented by the density matrix $P_{x} \varrho P_{x}+\left(1-P_{x}\right) \varrho\left(1-P_{x}\right) . P_{x} \varrho P_{x}$ is always proportional to $x \otimes \bar{x}$, irrespective of the choice of $\varrho$, and the constant of proportionality is the transition probability. If $\omega$ were not pure, we would not be able to find an observable with the required property. For this reason, and to preserve the symmetry between $\omega$ and $\omega^{\prime}$, we have defined the transition probability only between pure states. 


\section{The Superposition Principle}

In quantum mechanics the superposition principle is exemplified by the statement that, if $\phi_{1}$ and $\phi_{2}$ are normalizable solutions of the timedependent Schrödinger equation, then $\phi_{1}+\phi_{2}$, the superposition of $\phi_{1}$ and $\phi_{2}$, is also a normalizable solution. From a logical point of view, this statement may be resolved into a definition, an axiom, and an assertion. It defines "superposition" as a mathematical term, contains the implied axiom that if $\phi_{1}$ and $\phi_{2}$ taken at time $t$ each represent a state then so does $\phi_{1}+\phi_{2}$, and asserts that the time translation preserves superpositions, We shall investigate the form the superposition principle takes in the algebraic context and our discussion correspondingly consists of three parts.

We first turn to the definition of superposition. We must not expect to be able to superpose any two states. To show this, we again consider the example of a system with commutative discrete superselection rules whose existence in quantum field theory was demonstrated by WICK, WIGHTMAN and WIGNER [18]. If $x$ and $x^{\prime}$ are unit vectors representing states $\omega_{x}$ and $\omega_{x^{\prime}}$ lying in different sectors, $1 / \sqrt{2}\left(x-x^{\prime}\right)$ and $1 / \sqrt{2}\left(x+x^{\prime}\right)$ both represent the mixed state $1 / 2\left(\omega_{x}+\omega_{x^{\prime}}\right)$ rather than two different superpositions of $\omega_{x}$ and $\omega_{x^{\prime}}{ }^{4}$. Hence superposition is restricted to pure states taken from the same sector.

Let $\omega$ be a state in the sector $S_{\hat{\pi}}$ and let $I_{\omega}$ be the left ideal $\left\{A: \omega\left(A^{*} A\right)=0\right\}$ in $\mathcal{A}$. For any $A \notin I_{\omega}$, the linear functional $\omega_{A}$ defined by

$$
\omega_{A}(B)=\frac{\omega\left(A^{*} B A\right)}{\omega\left(A^{*} A\right)} \text { for all } B \in \mathfrak{A}
$$

is a pure state in $S_{\hat{x}}$, and, as we have seen in Proposition 4.2, any $\omega^{\prime} \in S_{\hat{\pi}}$ may be represented in this form [19]. Note that $\omega_{I}=\omega$ and $\left(\omega_{A}\right)_{B}=\omega_{B A}$ if $B \notin I_{\omega_{A}}$.

Definition 5.1. Let $\omega \in P(\mathfrak{A})$ and let $A_{1}, A_{2} \in \mathfrak{A}$ be linearly independent $\left(\bmod I_{\omega}\right)$, then $\omega_{A_{1}+A_{2}}$ is called a superposition of $\omega_{A_{1}}$ and $\omega_{A_{2}}$.

The connexion with the customary definition becomes clear when we pass to the Hilbert space formalism. $\pi_{\omega}\left(A_{1}\right) x_{\omega}$ and $\pi_{\omega}\left(A_{2}\right) x_{\omega}$ are linearly independent vectors of $\mathscr{H}_{\omega}$ representing $\omega_{A_{1}}$ and $\omega_{A_{2}}$ respectively and $\pi_{\omega}\left(A_{1}\right) x_{\omega}+\pi_{\omega}\left(A_{2}\right) x_{\omega}$ represents $\omega_{A_{1}+A_{2}}$. Definition 5.1 automatically rules out superpositions of pure states from different sectors and $\omega_{A_{1}+A_{2}}$ necessarily lies in the same sector as $\omega_{A_{1}}$ and $\omega_{A_{3}}$. It must be emphasized that $\omega_{A_{1}+A_{2}}$ depends on the particular choice of $\omega, A_{1}$ and $A_{2}$, and not merely on $\omega_{A_{1}}$ and $\omega_{A_{z}}$. We shall parametrize all possible superpositions and start with a simple Lemma.

4 A statistical mixture is sometimes referred to as a superposition and what is meant here is then called a coherent superposition. 
Lemma 5.2. Let $\omega \in P(\mathfrak{A})$ and $A, B \notin I_{\omega}$. Then $\omega_{A}=\omega_{B}$ if and only if $B=\lambda A\left(\bmod I_{\omega}\right)$ for some $\lambda \in \boldsymbol{C}, \lambda \neq 0$.

Proof. If $\lambda \in \boldsymbol{C}, \lambda \neq 0$ and $N \in I_{\omega}$, then $\omega_{\lambda A+N}=\omega_{A}$ by a simple application of the Cauchy-Schwarz inequality $[11 ; 2.1 .2]$. Conversely if $\omega_{A}=\omega_{B},\left\|\omega_{A}-\omega_{B}\right\|=0$ and

$$
\omega_{A} \cdot \omega_{B}=\frac{\left|\omega\left(A^{*} B\right)\right|^{2}}{\omega\left(A^{*} A\right) \omega\left(B^{*} B\right)}=1 .
$$

Hence $\omega\left((B-\lambda A)^{*}(B-\lambda A)\right)=0$ has a non-zero solution in $\lambda$.

This Lemma is actually just a consequence of the fact that $S_{\hat{\pi}_{\omega}}$ may be identified with $P\left(\mathscr{H}_{\omega}\right)$, the projective space of $\mathscr{H}_{\omega}$ and that $\mathscr{H}_{\omega}=\mathfrak{X} / I_{\omega}$ [19 and $11 ; 2.8 .5]$. It shows that, keeping $\omega, A_{1}$ and $A_{2}$ fixed, $\lambda \mapsto \omega_{A_{1}+\lambda A_{2}}$ $\lambda \in \boldsymbol{C}, \lambda \neq 0$, parametrizes all possible superpositions of $\omega_{A_{1}}$ and $\omega_{A_{2}}$. The triplet $\left(\omega, A_{1}, A_{2}\right)$ may always be chosen so that $A_{1}=I$ and $A_{2}=U$, where $U$ is unitary. Let us suppose that $\omega$ and $\omega_{U}$ are orthogonal, i.e. $\omega \cdot \omega_{U}=0$, and let us set $\lambda=\tan \theta e^{i \phi}, 0<\theta<\pi / 2,0 \leqq \phi<2 \pi$. Then

$$
\begin{aligned}
\omega_{I+\lambda U}(A)= & \cos ^{2} \theta \omega(A)+\sin ^{2} \theta \omega_{U}(A)+\cos \theta \sin \theta\left[e^{i \phi} \omega(A U)\right. \\
& +e^{-i \phi} \omega\left(U^{*} A\right)\left(^{*}\right)
\end{aligned}
$$

parametrizes all superpositions of $\omega$ and $\omega_{U}$. If we take the mean of the equation (*) with respect to the phase $\phi$, the "interference term" drops out leaving the mixed state $\omega^{\prime}=\cos ^{2} \theta \omega+\sin ^{2} \theta \omega_{V}$. Thus there is a mixed state $\omega^{\prime}$ with mixing ratio $|\lambda|^{2}=\tan ^{2} \theta$ corresponding to any superposition $\omega_{I+\lambda U}$.

A set $M$ of pure states of $\mathfrak{A}$ will be said to be closed under superpositions if it contains every superposition of any pair of its elements. If $M$ is not closed under superpositions, let $\Lambda(M)$ denote the smallest subset of $P(\mathfrak{R})$ closed under superpositions and containing $M$. For example if $M=\left\{\omega_{A_{i}}: i=1,2, \ldots, n\right\}$ where the $A_{i}, i=1,2, \ldots, n$, are linearly independent $\left(\bmod I_{\omega}\right)$, then the matrix $\omega\left(A_{i}^{*} A_{j}\right)$ is strictly positive and

$$
K=\left\{\left(\lambda_{1}, \lambda_{2}, \ldots, \lambda_{n}\right): \sum_{i, j} \bar{\lambda}_{i} \lambda_{i} \omega\left(A_{i}^{*} A_{j}\right)=1\right\}
$$

is a compact subset of $\boldsymbol{C}^{n}$ intersecting every ray through the origin. Any state $\omega^{\prime}$ of the form $\omega^{\prime}(B)=\sum_{i, j} \bar{\lambda}_{i} \lambda_{j} \omega\left(A_{i}^{*} B A_{j}\right)$ for all $B \in \mathcal{A}$, with $\left(\lambda_{1}, \lambda_{2}, \ldots, \lambda_{n}\right) \in K^{\prime}$ is an element of $\Lambda(M)$. The map $\phi: K \rightarrow \Lambda(M)$ so defined is onto and two $n$-tuplets are mapped onto the same state if and only if they differ by a common phase factor. In this way, we see that $\Lambda(M)$ is isomorphic to $P_{n-1}(\boldsymbol{C})$, the complex projective space of $n-1$ dimensions.

As we have seen, $P(\mathfrak{A})$ is the disjoint union of its sectors and each sector $S_{\hat{\pi}}$ may be regarded as the unit rays of a Hilbert space $\mathfrak{A} / I_{\omega}$, where $\omega \in S_{\hat{\pi}}$. This allows us to perform on $P(\mathfrak{Q})$ many of the operations 
associated with a Hilbert space. We have already seen this above in the case of the operation $\Lambda$ which corresponds to taking linear hulls. Another example is forming orthogonal complements: if $M \subset P(\mathfrak{A})$, we simply set $M L=\left\{\omega \in P(\mathfrak{U}): \omega \cdot \omega^{\prime}=0\right.$ for all $\left.\omega^{\prime} \in M\right\}$. We may also close $\Lambda(M)$ in the norm topology and, as in the linear case, we get $M \perp \perp$ $=\bar{\Lambda}(M)$. If $M \subset S_{\hat{\pi}}$, then $\bar{\Lambda}(M)$, as a metric space, is actually uniformly isomorphic to a projective Hilbert space. We can introduce the concept of an orthogonal basis in $P(\mathfrak{A})$ and prove the analogue of Bessel's inequality and Parseval's equation. We can also give an algebraic version of the Gram-Schmidt orthogonalization process by taking advantage of the representation of $S_{\hat{\pi}}$ using $\mathfrak{A} / I_{\omega}$.

We turn now to the structure of the physical states $\mathscr{S}$ of the system and the role of the superposition principle as an axiom on the structure of the pure physical states. Let $\mathscr{S}_{p}=\mathscr{S} \cap P(\mathfrak{U})$ and let us call a sector $S_{\hat{\boldsymbol{\pi}}}$ physical if $\mathscr{S}_{p} \cap S_{\hat{\boldsymbol{\pi}}} \neq \emptyset$. The physical spectrum of $\mathfrak{A}$ is then $\hat{\mathfrak{U}}_{p}=\left\{\hat{\pi} \in \hat{\mathfrak{A}}: S_{\hat{\pi}}\right.$ is physical $\}$. We can now formulate the

Superposition Principle: The superposition of two physical pure states is again a physical pure state. Thus $\Lambda\left(\mathscr{S}_{p}\right)=\mathscr{S}_{p}$.

If $S_{\hat{\pi}}$ is a physical sector, the superposition principle says that $S_{\hat{\boldsymbol{\lambda}}} \cap \mathscr{S}_{p}$ is closed under superpositions. The exact form of $S_{\hat{\boldsymbol{\pi}}} \cap \mathscr{S}_{p}$ is probably of little consequence; we assume that it is norm dense in $S_{\hat{\boldsymbol{\pi}}}$. It is often convenient to regard $S_{\hat{\pi}} \cap \mathscr{S}_{p}$ as the whole of $S_{\hat{\pi}}$, although a more intelligent guess would be to take it to be those states of $S_{\hat{x}}$ with a finite expectation value of the energy. We have already assumed that $\mathscr{S}$ is convex and we follow MAckex [2] in assuming that $\mathscr{S}$ is closed under forming countable convex combinations. It is consistent with the emphasis on pure states in this paper to suppose that $\mathscr{S}_{p}$ is the set of extreme points of $\mathscr{S}$. Thus

$$
\mathscr{S}=\left\{\sum_{i=1}^{\infty} \lambda_{i} \omega_{i}: \omega_{i} \in \mathscr{S}_{p}, 0 \leqq \lambda_{i}<1, \sum_{i=1}^{\infty} \lambda_{i}=1\right\} .
$$

Finally, we want to show that superpositions are preserved by symmetry transformations.

Proposition 5.3. If $M \subset P(\mathfrak{U})$ and $\alpha: S(\mathfrak{U}) \rightarrow S(\mathfrak{U})$ arises from a symmetry, then $\alpha(\Lambda(M))=\Lambda(\alpha(M))$.

Proof. It suffices to prove the result if $M$ is finite. As $\alpha$ is an affine automorphism by Proposition 3.1, $\alpha$ must leave $P(\mathfrak{U})$ invariant. However $\alpha$ is also an isometry, Proposition 3.2, so that $\alpha(M \perp)=\alpha(M)^{\perp}$. Hence $\alpha(\bar{\Lambda}(M))=\bar{\Lambda}(\alpha(M))$. But if $M$ is finite $\Lambda(M)=\bar{\Lambda}(M)$ giving the result.

This completes the three parts of our analysis of the superposition principle, for a time translation is merely a particular example of a symmetry. In conclusion, we add a few remarks on the physical meaning of superposition. The transition $\omega \longmapsto \omega_{A}$ is an example of a pure operation 
[5] and may be regarded as induced by a local measurement or some local "influence" transforming pure states into pure states. The superposition principle given above may be reformulated to read: if a state $\omega \in \mathscr{S}_{p}$ is acted on by influences $A_{1}$ and $A_{2}$, the resulting state is again in $\mathscr{S}_{p}$ and may be obtained by a single transition $\omega \longmapsto \omega_{A_{1}+A_{2}}$ corresponding to the sum of the influences. This corresponds to its usual formulation in classical physics. Actually from this point of view the purity of $\omega$ is irrelevant and Definition 5.1 could easily be modified accordingly.

\section{Wigner's Theorem and Symmetries}

We now take up our analysis of symmetries to deduce that a symmetry leaves transition probabilities invariant and permutes the sectors.

Proposition 6.1. If $\alpha: S(\mathfrak{U}) \rightarrow S(\mathfrak{U})$ arises from a symmetry and $\omega, \omega^{\prime} \in P(\mathfrak{U})$, then $\alpha(\omega) \cdot \alpha\left(\omega^{\prime}\right)=\omega \cdot \omega^{\prime}$. Further if $S$ is a sector then so is $\alpha(S)$ and $\alpha$ induces a bijection $\hat{\alpha}: \hat{\mathfrak{A}} \rightarrow \hat{\mathfrak{A}}$.

Proof. As $\alpha$ is an affine isomorphism by Proposition 3.1, $\alpha$ must leave $P(\mathfrak{U})$ invariant. However, by Proposition $3.2, \alpha$ is an isometry, so by Definition $4.7, \alpha(\omega) \cdot \alpha\left(\omega^{\prime}\right)=\omega \cdot \omega^{\prime}$. An isometry of $P(\mathfrak{A})$ must induce a permutation of the components of $P(\mathfrak{U})$ in the norm topology and by Proposition 4.5, these are just the sectors. Hence $\alpha$ induces a bijection $\hat{\alpha}: \hat{\mathfrak{A}} \rightarrow \hat{\mathfrak{A}}$.

Proposition 4.6 shows that our definition of transition probability is consistent with the usual one so we are in a position to apply Wigner's Theorem [8] to deduce the form of $\alpha$.

Proposition 6.2. If $\alpha: S(\mathfrak{U}) \rightarrow S(\mathfrak{A})$ arises from a symmetry and if $\pi$ and $\pi^{\prime}$ are irreducible representations of $\mathfrak{A}$ such that $\hat{\pi}^{\prime}=\hat{\alpha}(\hat{\pi})$ then there exists a mapping $W: \mathscr{H}_{\pi} \rightarrow \mathscr{H}_{\pi^{\prime}}$ linear with respect to the real numbers and unique to within a phase factor such that $\alpha\left(\omega_{x}\right)=\omega_{W x}$ for all unit vectors $x \in \mathscr{H}_{\pi} . W$ is either unitary or antiunitary.

Proof. The elements of $S_{\hat{\pi}}$ and $S_{\hat{\pi}^{\prime}}$ are in natural $1-1$ correspondence with the rays of the Hilbert spaces $\mathscr{H}_{\pi}$ and $\mathscr{H}_{\pi^{\prime}}$ respectively. Hence $\alpha$ induces a bijection of the rays of $\mathscr{H}_{\pi}$ onto those of $\mathscr{H}_{\pi^{\prime}}$. Applying Wigner's Theorem in the extended form due to BARGMANN [9], the assertion follows.

We can also characterize $\bar{\alpha}: \mathfrak{A} \rightarrow \mathfrak{A}$ and obtain thereby a result due to Kadison [10; Corollary 4.7].

Proposition 6.3. If $\bar{\alpha}: \mathfrak{A} \rightarrow \mathfrak{A}$ arises from a symmetry and $A \in \mathfrak{A}$ then $\bar{\alpha}(A)^{2}=\bar{\alpha}\left(A^{2}\right)$. In other words $\bar{\alpha}$ is a Jordan homomorphism and, since $\bar{\alpha}$ is a bijection, $\bar{\alpha}$ is in fact a Jordan isomorphism.

\footnotetext{
5 A trivial modification is necessary if $\mathscr{H}_{\pi}$ is one-dimensional.
} 
Proof. With the notation of Proposition 6.2, if $x \in \mathscr{H}_{\pi}$ is a unit vector, $(x, \pi(A) x)=\omega_{x}(A)=\alpha\left(\omega_{x}\right)(\bar{\alpha}(A))=\left(W_{x}, \pi^{\prime} \circ \bar{\alpha}(A) W x\right)$. Suppose for definiteness that $W$ is antiunitary, then

$$
(x, \pi(A) x)=\left(x,\left(W^{-1} \pi^{\prime} \circ \bar{\alpha}(A) W\right)^{*} x\right)
$$

for all $x \in \mathscr{H}_{\pi}$ and thus $(x, \pi(A) y)=\left(x,\left(W^{-1} \pi^{\prime} \circ \bar{\alpha}(A) W\right)^{*} y\right)$ for all $x, y \in \mathscr{H}_{\pi}$. Hence $\pi^{\prime} \circ \bar{\alpha}(A)=W \pi\left(A^{*}\right) W^{-1}$ and so

$$
\pi^{\prime}\left(\bar{\alpha}(A)^{2}\right)=W \pi\left(A^{2 *}\right) W^{-1}=\pi^{\prime} \circ \bar{\alpha}\left(A^{2}\right) .
$$

If $W$ is unitary, we obtain $\pi^{\prime} \circ \bar{\alpha}(A)=W \pi(A) W^{-1}$ and again $\pi^{\prime}\left(\bar{\alpha}(A)^{2}\right)$ $=\pi^{\prime} \circ \bar{\alpha}\left(A^{2}\right)$. Hence $\bar{\alpha}\left(A^{2}\right)=\bar{\alpha}(A)^{2}$ since a $C^{*}$-algebra has enough irreducible representations $[11 ; 2.7 .3]$. Thus $\bar{\alpha}$ is a Jordan isomorphism.

It is a matter of simple manipulation to deduce that $\bar{\alpha}(A B+B A)$ $=\bar{\alpha}(A) \bar{\alpha}(B)+\bar{\alpha}(B) \bar{\alpha}(A)$ for all $A, B \in \mathfrak{Q}$. This is perhaps more familiar as the definition of a Jordan homomorphism ${ }^{6}$.

It is natural to ask whether, if we are given a bijection $\hat{\alpha}: \hat{\mathfrak{A}} \rightarrow \hat{\mathfrak{Q}}$, a representation $\pi_{i}$ of $\mathfrak{A}$ in $\mathscr{H}_{i}$ for each $i \in \hat{\mathfrak{A}}$ and a unitary or antiunitary map $W_{i}: \mathscr{H}_{i} \rightarrow \mathscr{H}_{\hat{\alpha}(i)}$, we may construct a pair of mappings $\alpha: S(\mathfrak{A}) \rightarrow S(\mathfrak{A}), \bar{\alpha}: \mathfrak{A} \rightarrow \mathfrak{A}$ having the properties of a symmetry such that $\alpha\left(\omega_{x}\right)=\omega_{W_{i} x}$ for all unit vectors $x \in \mathscr{H}_{i}$. This is not in general possible, and to obtain a result with a converse, we must first strengthen Proposition 6.2.

Proposition 6.4. If $\alpha: S(\mathfrak{A}) \rightarrow S(\mathfrak{A})$ arises from a symmetry, let $\pi=\oplus \pi_{i} ; \pi$ is called the reduced atomic representation. Then there exists a mapping $W: \mathscr{H}_{\pi} \rightarrow \mathscr{H}_{\pi}$, linear with respect to the real numbers, such that $\alpha\left(\omega_{x}\right)=\omega_{W x}$ for all unit vectors $x \in \mathscr{H}_{\pi}$. If $W^{\prime}$ is any other such mapping, $W^{\prime}=W \bigoplus_{i \in \hat{\mathscr{U}}} e^{i \lambda_{i}} I_{i}$, where $I_{i}$ is the identity operator on $\mathscr{H}_{i}$ and $\lambda_{i}$ is real.

In other words, $W$ is unique ${ }^{7}$ to within a phase factor on each $\mathscr{H}_{i}$. Further $W$ has the following properties:
a) $|(W x, W y)|=|(x, y)|$
b) $W\left(\mathscr{H}_{i}\right) \quad=\mathscr{H}_{\hat{\alpha}(i)}$ for all $i \in \hat{\mathfrak{Q}}$
c) $W \pi\left(\mathfrak{A}_{h}\right) W^{-1}=\pi\left(\mathfrak{A}_{h}\right)$.

Proof. If $x \in \mathscr{H}_{i}$ is a unit vector, $\omega_{x} \in S_{i}$ and $\alpha\left(\omega_{x}\right) \in S_{\hat{\alpha}(i)}$. Hence if $\alpha\left(\omega_{x}\right)=\omega_{y}, y$ must be a unit vector of $\mathscr{H}_{\hat{\alpha}(i)}$. Thus $W$, if it exists, must satisfy b) and by Proposition 6.2 must coincide on $\mathscr{H}_{i}$ with a unitary or antiunitary operator $W_{i}: \mathscr{H}_{i} \rightarrow \mathscr{H}_{\hat{\alpha}(i)}$. Thus $W$ must satisfy $(W x)_{\hat{\alpha}(i)}$ $=W_{i} x_{i}$ for all $x \in \mathscr{H}_{\pi}$, where $x_{i}$ denotes the projection of $x$ onto $\mathscr{H}_{i}$. However, if we use this equation to define a $W, W$ is automatically linear with respect to the real numbers and satisfies a) and b). Now if

6 The term used by KaDISON [10] is $C^{*}$-homomorphism.

7 A trivial modification is again necessary if some of the $\mathscr{H}_{i}$ are one-dimensional. 
$x \in \mathscr{H}$ is a unit vector $\omega_{x}=\Sigma_{i}^{\prime}\left\|x_{i}\right\|^{2} \omega_{y_{i}}$ where $y_{i}=x_{i} /\left\|x_{i}\right\|$ and the sum is taken over all $i \in \hat{\mathfrak{U}}$ such that $x_{i} \neq 0$. Hence $\alpha\left(\omega_{x}\right)=\Sigma_{i}^{\prime}\left\|x_{i}\right\|^{2} \alpha\left(\omega_{y_{i}}\right)$, and $\alpha\left(\omega_{x}\right)=\omega_{W x}$ for all unit vectors $x \in \mathscr{H}_{\pi}$. It remains to show that $W$ satisfies c). Now from the proof of Proposition 6.3, $\pi_{\hat{\alpha}(i)} \circ \bar{\alpha}(A)$ $=W_{i} \pi_{i}(A) W_{i}^{-1}$ if $A \in \mathfrak{A}_{h}$. A routine calculation now shows that $\pi \circ \bar{\alpha}(A)=W \pi(A) W^{-1}$, which implies c). The statement on the degree of uniqueness of $W$ follows from the construction and Proposition 6.2.

Proposition 6.5. Let $\pi$ be the reduced atomic representation. Given a mapping $W: \mathscr{H}_{\pi} \rightarrow \mathscr{H}_{\pi}$ which is linear with respect to the real numbers and satisfies:

a) $|(W x, W y)|=|(x, y)|$ for $x, y \in \mathscr{H}_{\pi}$.

b) $W\left(\mathscr{H}_{i}\right)=\mathscr{H}_{\hat{\alpha}(i)}$ where $\hat{\alpha}: \hat{\mathfrak{Q}} \rightarrow \hat{\mathfrak{Q}}$ is a bijection.

c) $W \pi\left(\mathfrak{A}_{h}\right) W^{-1}=\pi\left(\mathfrak{A}_{h}\right)$.

Then the exist unique bijections $\alpha: S(\mathfrak{A}) \rightarrow S(\mathfrak{U}), \bar{\alpha}: \mathfrak{A} \rightarrow \mathfrak{A}$ such that $\mu(\alpha(\omega), \bar{\alpha}(A))=\mu(\omega, A)$ for $\omega \in S(\mathfrak{A})$ and $A \in \mathfrak{A}_{h}$ and $\alpha\left(\omega_{x}\right)=\omega_{W x}$ for all unit vectors $x \in \mathscr{H}_{\pi}$.

Proof. The reduced atomic representation $\pi$ is faithful since a $C^{*}$. algebra has sufficient irreducible representations and we may use c) to define a mapping $\bar{\alpha}: \mathfrak{A}_{h} \rightarrow \mathfrak{A}_{h}$ by setting $\pi \circ \bar{\alpha}(A)=W \pi(A) W^{-1}$. Further $\bar{\alpha}$ is isometric, onto and real linear since $W$ has these properties. $\bar{\alpha}$ extends to a linear mapping $\bar{\alpha}: \mathfrak{A} \rightarrow \mathfrak{A}$ which is also an isometry and onto as may be shown, for example, by using the inequalities $\max (\|A\|$, $\|B\|) \leqq\|A+i B\| \leqq\|A\|+\|B\|$ valid for $A, B \in \mathfrak{A}_{h}$. $\alpha$ must now be defined as the restriction to $S(\mathfrak{Q})$ of the dual of $\bar{\alpha}^{-1}$, so as to satisfy $\alpha(\omega)(\bar{\alpha}(A))=\omega(A)$. If $x \in \mathscr{H}_{\pi}$ is a unit vector and $A \in \mathfrak{A}_{h}$,

$$
\alpha\left(\omega_{x}\right)(\bar{\alpha}(A))=(x, \pi(A) x)=(W x, \pi \circ \bar{\alpha}(A) W x)=\omega_{W x}(\bar{\alpha}(A)) .
$$

Hence $\alpha\left(\omega_{x}\right)=\omega_{W x}$ as required. If $A \in \mathfrak{A}_{h}$ and $g$ is a continuous function on the spectrum of $A$, then $\bar{\alpha}(g(A))=g(\bar{\alpha}(A))$ since $\bar{\alpha}$ induces an isomorphism of the commutative subalgebra of $\mathfrak{A}$ generated by $A$ onto that generated by $\bar{\alpha}(A)$. Hence $\mu(\alpha(\omega), \bar{\alpha}(A))=\mu(\omega, A)$ for $\omega \in S(\mathfrak{A})$ and $A \in \mathfrak{A}_{h}$. Now $\alpha: P(\mathfrak{U}) \rightarrow P(\mathfrak{U})$ is uniquely determined by the conditions of the Proposition, hence $\bar{\alpha}: \mathfrak{A} \rightarrow \mathfrak{A}$ is uniquely determined since we must have $\alpha(\omega)(\bar{\alpha}(A))=\omega(A)$. Thus $\alpha: S(\mathfrak{U}) \rightarrow S(\mathfrak{U})$ is also uniquely determined completing the proof of the Proposition.

There may still be no symmetry of the physical system which gives rise to the mappings $\alpha$ and $\bar{\alpha}$ of Proposition 6.5 because, in the analysis of concrete physical systems, there may be physical reasons, for example uniqueness and invariance of the vacuum, placing further restrictions on the nature of $\alpha$ and $\bar{\alpha}$. Even within the framework of this paper, we have not quite completed our analysis because we assumed that $\alpha(\mathscr{S})=\mathscr{S}$. However, with the assumption and notations of the previous section, we can easily fill this gap. Let $\pi^{\prime}=\bigoplus_{i \in \hat{\vartheta}_{p}} \pi_{i}$ then each $\omega \in \mathscr{S}_{p}$ is represented
23 Commun. math.Phys.,Vol.11 
by a unit vector of $\mathscr{H}_{\pi^{\prime}}$ and each $\omega \in \mathscr{S}$ by a density matrix of $\mathscr{H}_{\pi^{\prime}}$. $\pi^{\prime}$ must be faithful since $\mathscr{S}$ is full and further $\hat{\alpha}\left(\hat{\mathfrak{V}}_{p}\right)=\hat{\mathfrak{A}}_{p}$. The analogues of Propositions 6.4 and 6.5 now hold with $\pi^{\prime}$ replacing $\pi . \alpha(\mathscr{S})=\mathscr{S}$ if and only if $W$ leaves the subspace spanned by $\left\{x \in \mathscr{H}_{\pi^{\prime}}:\|x\|=1\right.$ and $\left.\omega_{x} \in \mathscr{S}_{p}\right\}$ invariant.

Acknowledgements. One of us (J.E.R.) wishes to thank The Royal Society for a research fellowship under their European Programme.

\section{References}

1. Neumann, J. von: Mathematische Grundlagen der Quantenmechanik. Berlin: Springer 1932.

2. Mackey, G. W.: Mathematical foundations of quantum mechanics. New York: Benjamin 1963.

3. Segal, I.: Postulates for general quantum mechanics. Ann. Math. 48, 930-948 (1947).

4. Arakr, H.: Lecture Notes (Zürich 1962) unpublished.

5. HAaG, R., and D. Kastler: An algebraic approach to field theory. J. Math. Phys. 5, 848-861 (1964).

6. Plymen, R. J.: $C^{*}$-algebras and Mackey's axioms. Commun. Math. Phys. 8, 132-146 (1968).

7. Davies, E. B.: On the Borel structure of $C^{*}$-algebras. Commun. Math. Phys. 8, 147-163 (1968).

8. Wigner, E. P.: Gruppentheorie, pp. 251-254. Braunschweig: Frederick Vieweg u. Sohn 1931; Group theory, pp. 233-236, New York: Academic Press 1959.

9. Bargmane, V.: Note on Wigner's theorem on symmetry operations. J. Math. Phys. 5, 862-868 (1964).

10. Kadison, R. V.: Transformations of states in operator theory and dynamics. Topology 3, Suppl. 2, 177-198 (1965).

11. Drxmier, J.: Les $C^{*}$-algèbres et leurs répresentations. Paris: Gauthier-Villars 1964.

12. Bourbaki, N.: Topologie générale, chap IX, 2nd. ed. Paris: Hermann 1958.

13. - Topologie générale, chap. I, 3rd. ed., Paris: Hermann 1961.

14. GLimm, J., and R. V. Kadison: Unitary operators in $C^{*}$-algebras. Pacific J. Math. 10, 547-556 (1960).

15. Kaplansky, I.: A theorem on rings of operators. Pacific J. Math. 1, 227-232 (1951).

16. Dixmier, J.: Les algèbres d'opérateurs dans l'espace hilbertien. Paris: GauthierVillars 1957.

17. Schatten, R.: Norm ideals of completely continuous operators. BerlinGöttingen-Heidelberg: Springer 1960.

18. Wick, G. C., A. S. Wightman, and E. P. Wigner: The intrinsic parity of elementary particles. Phys. Rev. 88, 101-105 (1952).

19. Kadison, R. V.: Irreducible operator algebras. Proc. Natl. Acad. Sci. US. 43, $273-276$ (1957).

J. E. Roberts

G. ROEPSTORFF

II. Inst. f. Theoret. Physik d. Universität 2000 Hamburg 50, Luruper Chaussee 149 